\title{
THE STELLAR TEMPERATURE SCALE FROM O5 TO AO
}

\author{
D. S. HA YES \\ Dept. of Physics and Astronomy, Rensselaer Polytechnic Institute, Troy, N.Y., U.S.A.
}

\begin{abstract}
An absolute spectrophotometric calibration of Vega and eleven other standard stars was completed by the author in 1967. This calibration extends from 3200 to $10870 \AA$, and therefore gives a useful calibration of the Paschen discontinuity as well as the Balmer discontinuity, for which calibrations have been available for some time.

The measured values of the ratio of the size of the Paschen discontinuity to the size of the Balmer discontinuity have been compared with corresponding values predicted by model atmospheres. The comparison shows that LTE models satisfactorily predict the continuum in the wavelength range given above.

A stellar temperature scale has been determined by fitting the measured sizes of the Balmer discontinuity of 43 stars to blanketed model atmospheres. This temperature scale agrees very well with Morton's modified form of the Morton-Adams temperature scale for all except the latest B-stars, for which higher temperatures are predicted.
\end{abstract}

\section{Introduction}

In 1967 the author completed a recalibration of the energy distribution of Vega (Hayes, 1967, 1969). It has been shown (Wolff et al., 1968, hereafter referred to as $\mathrm{WKH}$ ) that this new calibration allows the determination of the temperatures of early-type stars through measurement of the Balmer discontinuity. That paper included stars extending only as early as B3V, and used unblanketed LTE model atmospheres. This paper reports a rediscussion of the WKH approach with the addition of a number of earlier stars, extending the scale to O5, and with the use of the blanketed model atmospheres calculated by the Princeton group (Mihalas and Morton, 1965; Adams and Morton, 1968; Hickok and Morton, 1968; Bradley and Morton, 1969).

In view of the recent discussion about the possible importance of departures from LTE on the Lyman continuum of model atmospheres, it should be made clear that a basic assumption of this paper is that the LTE blanketed models are correct. This means that ' $T_{\mathrm{e}}$ ' ( or $\theta_{\mathrm{e}}=5040 / T_{\mathrm{e}}$ ) will be here taken to be a parameter defined by the LTE models. The physical significance of this parameter may still be open to some re-interpretation once accurate non-LTE models become available.

\section{Observations}

The 43 stars used in this temperature scale have been selected from scanner observations by the author (the data from 3200 to $7350 \AA$ previously published in WKH), from the 13-color photometry by Mitchell and Johnson (1969), and from the list of scanner observations by Whiteoak (1966). In the latter two cases, the observations have been reduced to the calibration of Vega by the author (Hayes, 1967, 1970). Further, they have been selected to represent single, normal near main-sequence stars, 
as much as possible. Exceptions are found in the O-stars, some of which are multiple. Of stars have been excluded. That the stars are single means that double-lined spectroscopic binaries and visual doubles with $\Delta \mathrm{m}<5^{\mathrm{m}} .0$ and a separation less than $1^{\prime}$ have been avoided. Among the stars later than B0, only little-reddened stars have been used.

\section{Interpretation of the Observations}

The quantity $B$ is defined as being $-2.5 \log \left[F\left(1 / \lambda=2.74^{+}\right) / F\left(1 / \lambda=2.74^{-}\right)\right]$, a measure of the Balmer discontinuity which is directly comparable with the models. It is to be distinguished from $D$, defined by Barbier and Chalonge (Barbier, 1955), which is $\log \left[F\left(1 / \lambda=2.7^{-}\right) / F\left(1 / \lambda=2.7^{+}\right)\right]$. It is also distinguished from $D$ by the method of

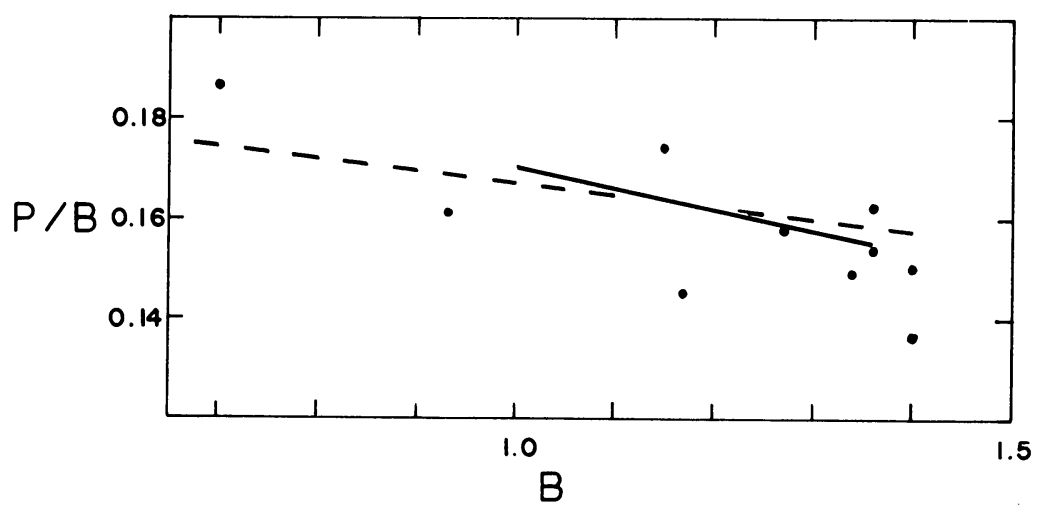

Fig. 1. The ratio of the logarithmic sizes of the Paschen and Balmer discontinuities plotted with respect to the Balmer discontinuity. The dots represent the observations. The dashed line is the locus of LTE models by Mihalas, and the solid line the locus of non-LTE models by Mihalas.

extrapolating to the wavelength of reference from longer wavelengths. A model atmosphere is selected which best fits the Paschen continuum from 4000 to $8000 \AA$. The model then allows a reproduceable extrapolation to $1 / \lambda=2.74^{-}$. Recall that $D$, however, uses a linear extrapolation from the blue region, with a much greater chance of errors of judgment. The lower point $\left(2.74^{+}\right)$is defined by the observations.

The quantity $B_{c}$ is simply the Balmer discontinuity of the model above, best fitting the Paschen continuum. Finally, $P=-2.5 \log \left[F\left(1 / \lambda=1.22^{+}\right) / F\left(1 / \lambda=1.22^{-}\right)\right]$is measured differentially with respect to the model.

Because of the linearity and range of photoelectric observations, we should not expect to find any errors in the measured values of $B$ which are a function of spectral type; the only systematic error present should be constant with spectral type and be due only to the calibration of Vega.

The calibration of the Paschen discontinuity allows an evaluation of the success of the LTE models in predicting the visual spectrum. Figure 1 shows the ratio $P / B$ plotted against $B$. Also shown are the corresponding results for LTE models by Mihalas (1964) 
and non-LTE models by Mihalas (1968). The non-LTE results of Mihalas indicate that the departures from LTE must be small in the second, third, and fourth levels of hydrogen. The observations confirm this. Thus, theory and observations agree that LTE models satisfactorily predict the stellar continuum in the region from 3200 to $11000 \AA$. If the new results reported by Oke and Schild at this symposium (this volume p. 13-17) are taken to indicate a larger value of $P$, the points in the diagram will move up, but by no more than 0.02 or at most 0.03 in $P / B$, which will not vitiate our conclusion.

The value of $B$ for Vega, that is the primary calibration, has been changed for this investigation by 0.04 , in the sense of making $B$ smaller. This has been done for several reasons, most arising because, in essence, we are performing an interpolation among other, more fundamental, temperature determinations. In particular, the size of the change, 0.04 , has been obtained by comparison with the results from $\mathrm{O}$-stars reported by Morton at this symposium (this volume, p. 59-63).

The reasons for the change are as follows. One is that the calibrations by Bahner (1963), Divan (1966) and the new results of Oke and Schild indicate that $B$ for Vega should be smaller by about this amount in the former two cases, and more in the latter case. Another reason is that only if $B$ is decreased by about 0.03 do we obtain agreement between $B$ and $B_{c}$; that is, the model which best fits the Paschen continuum should have a value of $B_{c}$ which is the same as the measured value of $B$ for the star.

A third reason for reducing $B$ for Vega arises because the temperatures are only defined with respect to the LTE models. The largest value of $B$ predicted by the models is about 1.40 at $\theta_{\mathrm{e}}=0.530$ for $\log g=4.0$. The calibration of Vega itself gives $B=1.44$, and the same value for $\theta$ Vir. It is not possible to fit these values of $B$ using lower gravities and still have the Paschen continuum fit the lower gravity model. Thus, the calibration and the models are fundamentally incompatible. By reducing $B$ of Vega of 0.04 , we obtain agreement. Otherwise, one should not even use these models for determining temperatures, because they would be undefined near A0.

As the classification parameter we have used the color $(U-V)$, instead of the MK type because rotation changes the apparent $T_{\mathrm{e}}$, without changing the $\mathrm{MK}$ type seriously (Collins, 1968), thus giving a spread in $T_{\mathrm{e}}$ at a given $\mathrm{MK}$ type. Increasing rotation causes a hypothetical star to move along the mean $T_{\mathrm{e}}$ vs. color line. The color $(U-V)$ is probably the best to use for early type stars because of its long baseline.

\section{Auxiliary Color Systems}

Since only MK types are available for the O-stars from Whiteoak's list, and since they are all reddened to some degree, it was necessary to find some means to obtain their unreddened colors. It was hoped that an unreddened color for each star could be obtained, rather than depending upon the mean intrinsic colors published by Johnson (1966). In order to obtain the maximum sensitivity to reddening, an auxiliary monochromatic color system was defined. The magnitudes $U V, G$, and $I R$ represent the scanner points at $1 / \lambda=2.80,2.02$, and 1.136 , respectively. For stars taken from Mit- 
chell and Johnson's 13-color photometry, the following transformations were used:

$$
\begin{aligned}
& (U V-G)=1.03(\mathbf{3 5})-0.03(\mathbf{3 3})-0.04(\mathbf{4 5}) \\
& (G-I R)=-0.65+0.935(\mathbf{5 2}-\mathbf{8 6})
\end{aligned}
$$

where $33,35, \mathbf{4 5}, \mathbf{5 2}$, and 86 denote 5 of the magnitudes in the 13-color system.

A color-color diagram was plotted for the stars, with the reddening line defined by five 08 stars with nearly the same value of $B$. The unreddened main sequence was defined by the models by Mihalas (1964). The unreddened value $(U V-G)_{0}$ was obtained for each star. A relation between $(U V-G)$ and $(U-V)$ was established by determining $(U V-G)$ for a large number of early-type stars for which thirteen color photometry was available. All of these stars also had been observed in the UBV sys- tem (Johnson et al., 1966). $(U V-G)$ was plotted vs. $(U-V)$ for 78 stars and the mean transformation line determined. With this transformation line the values of $(U-V)_{0}$ for all of the program stars was obtained. It should be noted that, based upon only a small sample, mean intrinsic colors for the O-stars could be determined, and they differ from those published by Johnson (1966).

\section{The Temperature Scale}

The values of $B$ for each star can be associated with values of the parameter $T_{\mathrm{e}}$ through the blanketed LTE models of the Princeton group. (In practice, the unblanketed LTE models by Mihalas (1964) were used, and the resulting values of $T_{\mathrm{e}}$ corrected to agree

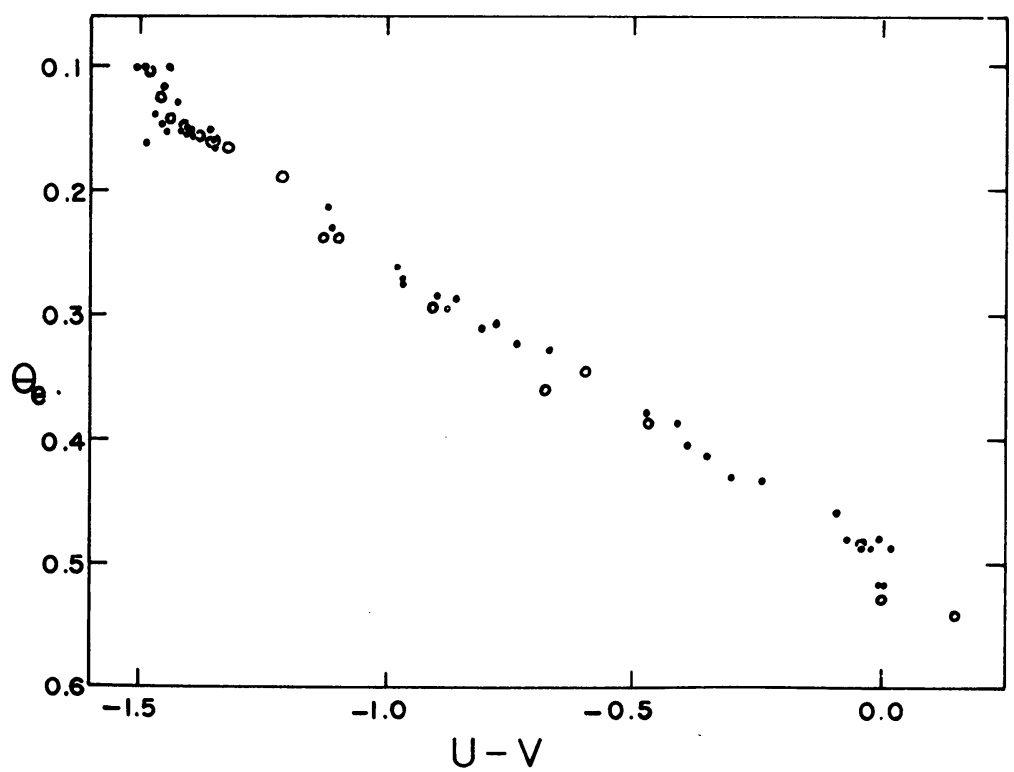

Fig. 2. The value of $\theta_{\mathrm{e}}=5040 / T_{\mathrm{e}}$ plotted with respect to $(U-V)$. The dots represent the observations from this paper. The circles for $(U-V)<-.1 .25$ represent the results by Morton, and those for $(U-V)>-1.25$ the Sidney interferometer results. 
with the blanketed models.) In Figure 2 are shown the values of $\theta_{\mathrm{e}}$ plotted vs. $(U-V)_{0}$. Also shown are the Sidney interferometer results (Brown et al., 1967). The low point at $(U-V)=-0.82$ is $\alpha$ Eri. It is discrepant probably because of an erroneous measurement of $(U-B)$. Morton's results are shown plotted with respect to the mean intrinsic colors for the early spectral types derived above from the auxiliary color system. It is quite clear that the present method has given temperatures in excellent agreement with those obtained by the other two techniques.

A mean line has been drawn through the points shown in Figure 2; it is shown in Figure 3. The corresponding values are listed in Table I. Although spectral types are given in this table for convenience, it is to be remembered that the identification of a color with a spectral type is to be regarded with great caution. Also shown in Figure 3

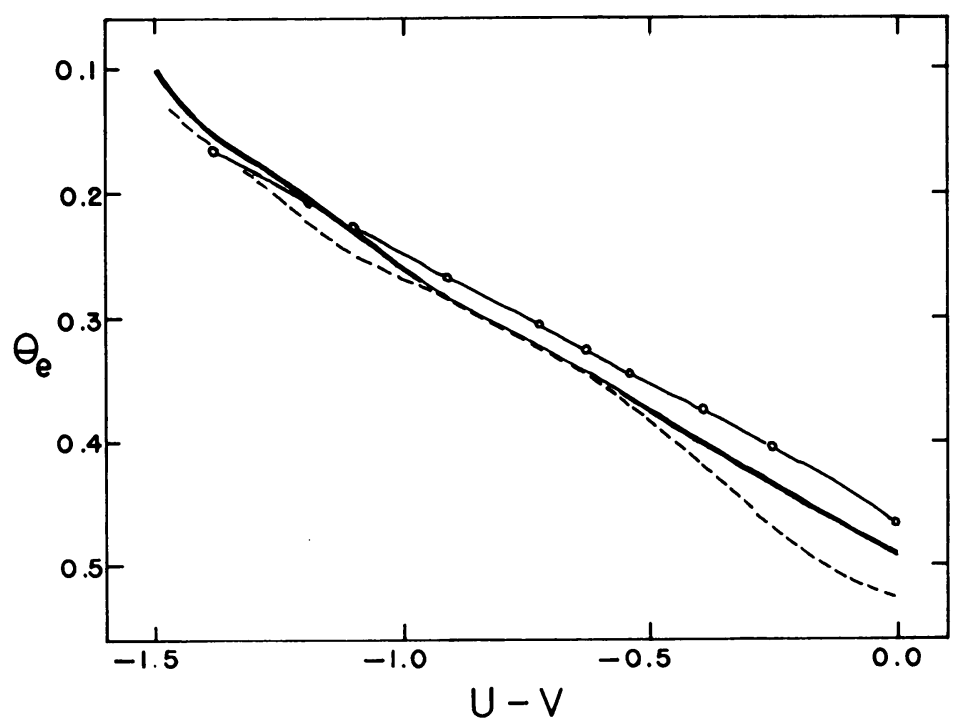

Fig. 3. The mean line derived from the data shown in Figure 2 (solid line). Also shown are the Morton-Adams temperature scale (dashed line) and that by Harris (line with circles).

TABLE I

The temperature scale

\begin{tabular}{lllllllll} 
MK Type $(U-V)$ & $\theta_{\mathrm{e}}$ & $T_{\mathrm{e}}$ & MK Type & $(U-V)$ & $(B-V)$ & $\theta_{\mathrm{e}}$ & $T_{\mathrm{e}}$ \\
& & & & & & & & \\
O5 & -1.48 & 0.112 & 45000 & B1 & -1.19 & -0.26 & 0.204 & 24700 \\
O6 & -1.46 & 0.123 & 40900 & B2 & -1.10 & -0.24 & 0.228 & 22100 \\
O7 & -1.44 & 0.133 & 37900 & B3 & -0.91 & -0.20 & 0.285 & 17700 \\
O8 & -1.41 & 0.145 & 34700 & B5 & -0.72 & -0.16 & 0.324 & 15600 \\
O9 & -1.38 & 0.154 & 32700 & B6 & -0.63 & -0.14 & 0.344 & 14700 \\
O9.5 & -1.35 & 0.163 & 30900 & B7 & -0.54 & -0.12 & 0.364 & 13800 \\
B0 & -1.32 & 0.170 & 29600 & B8 & -0.39 & -0.09 & 0.401 & 12600 \\
B0.5 & -1.28 & 0.180 & 28000 & B9 & -0.25 & -0.06 & 0.435 & 11600 \\
& & & & A0 & 0.00 & 0.00 & 0.492 & 10200 \\
\hline
\end{tabular}


are the temperature scales by Harris (1963) and Morton and Adams (1968). It is wellknown that the temperatures given by Harris are too high in the middle and later $B$ stars and this is clearly shown here. The agreement with the Morton-Adams temperatures is good in the earliest stars, excellent in the middle B-stars, but poor for the late B stars. The present temperatures should be superior to the Morton-Adams temperatures because the present ones rely upon photoelectric measurements of $B$, whereas the latter depend upon the older photographic spectrophotometry by Chalonge and Divan (1952).

A comparison of the observed ultraviolet fluxes with those predicted by models has already been given by Bless in his review paper at this symposium (this volume, p. 73) and also by Bless et al. (1968). Both of these papers refer to the Morton-Adams

- temperature scale. If we refer to the differences between the temperature scales cited above, we can conclude that for the early B stars the UV fluxes agree very well with model predictions when the present temperature scale is used. But for the late B stars, the observed fluxes fall significantly below the models.

\section{Acknowledgement}

I would like to thank D. C. Morton for allowing me to quote his results in advance of publication.

\section{References}

Adams, T. F. and Morton, D. C.: 1968, Astrophys. J. 152, 195.

Bahner, K.: 1963, Astrophys. J. 138, 1314.

Barbier, D.: 1955, 'Principes fondamentaux de classification stellaire', Colloques Int. Centre Nat. Rech. Sc., Paris, 55, 47.

Bless, R. C., Code, A. D., and Houck, T. E.: 1968, Astrophys. J. 153, 561.

Bradley, P. T. and Morton, D. C.: 1969, Astrophys. J. 156, 687.

Brown, R. Hanbury, Davis, J., Allen, L. R., and Rome, J. M.: 1967, Monthly Notices Roy. Astron. Soc. 137, 393.

Chalonge, D. and Divan, L.: 1952, Ann. Astrophys. 15, 201.

Collins II, G. W.: 1968, Astrophys. J. 152, 847.

Divan, L.: 1966, IAU Symposium 24: Spectral Classification and Multicolour Photometry (ed. by K.Loden, L. O. Loden, and U. Sinnerstad), Academic Press, London and New York, p. 311.

Harris III, D. L.: 1963, 'The Stellar Temperature Scale and Bolometric Corrections' in Stars and Stellar Systems, Vol. III, Basic Astronomical Data (ed. by K. Aa. Strand), University of Chicago Press, Chicago, p. 263.

Hayes, D. S.: 1967, Unpublished Ph.D. dissertation, University of California at Los Angeles.

Hayes, D. S.: 1970, Astrophys. J. 159, 165.

Hickok, F. R. and Morton, D. C.: 1968, Astrophys. J. 152, 203.

Johnson, H. L.: 1966, Ann. Rev. Astron. Astrophys. 4, 193.

Johnson, H. L., Mitchell, R. I., Iriarte, B., and Wisniewski, W. Z.: 1966, Comm. Lun. Planet. Lab. 4, 99.

Mihalas, D.: 1964, Astrophys. J. Suppl. Ser. 9, 321.

Mihalas, D.: 1968, Astrophys. J. 153, 317.

Mihalas, D. M. and Morton, D. C.: 1965, Astrophys. J. 142, 253.

Mitchell, R. I. and Johnson, H. L.: 1969, Comm. Lun. Planet. Lab. 8, 1.

Morton, D. C. and Adams, T. F.: 1968, Astrophys. J. 151, 611.

Whiteoak, J. B.: 1966, Astrophys. J. 144, 305.

Wolff, S. C., Kuhi, L. V., and Hayes, D.: 1968, Astrophys. J. 152, 871. 


\section{Discussion}

Houziaux: What is the reason for defining quantities such as $B, B_{c}, P$ etc., which in fact refer to quantities that do not exist? There are no such things as 'discontinuities'. What you have is a lot of close lines which overlap and gradually merge to reach the continuous spectrum on the short wavelength side of the 'discontinuity'. In certain cases, especially when the 'discontinuities' are small, such an extrapolation may be an additional source of error. Is it not just as good to take the flux ratio at two points where we believe we see the true continuum?

Hayes: Such a flux ratio would be sensitive not only to the size of the Balmer discontinuity, for example, but also to contributions to the slope of the continuum, such as errors in the calibration or interstellar reddening. I wish to isolate the effects of the Balmer discontinuity from those of the slope.

Kuhi: Does this mean that you are changing the absolute calibration again? And if so, what went wrong with the original experiment?

Hayes: Not really. Remember that I am using this technique as a form of interpolating device between the temperatures given by Morton at the high end and those from the Sidney interferometer results at the other end. I also want to emphasize that the temperature scale is constructed with the LTE models, and that one reason for changing the value of $B$ consistently by $0 .{ }^{\mathrm{m}} 04$ is to make the observations and the models compatible at the low end. I am not recommending this as a general change in my calibration, although it is within the range of the normal errors of measurement of that calibration.

Martynov: The use of symbol $T_{\mathrm{e}}$ for the temperature of the star determined from the measures of outgoing flux only is misleading because $T_{\mathrm{e}}$ usually denotes the effective temperature which can be determined only for stars with known dimensions and distance (Sun and the components of some eclipsing binaries), or stars with known angular diameters. The temperature discussed in the paper by Hayes (as well as by Bless) is 'colour temperature', or 'distribution temperature'.

Hayes: This use of the symbol ' $T \mathrm{e}$ ' is an interpretation with respect to the models. Its relation to a physical effective temperature depends upon the accuracy of the models. It is an effective temperature, however, because it depends upon the total radiation predicted by the model. 